\title{
THE DISCRETE LOGARITHM PROBLEM IN BERGMAN'S NON-REPRESENTABLE RING
}

\author{
MATAN BANIN AND BOAZ TSABAN
}

\begin{abstract}
Bergman's Ring $E_{p}$, parameterized by a prime number $p$, is a ring with $p^{5}$ elements that cannot be embedded in a ring of matrices over any commutative ring. This ring was discovered in 1974. In 2011, Climent, Navarro and Tortosa described an efficient implementation of $E_{p}$ using simple modular arithmetic, and suggested that this ring may be a useful source for intractable cryptographic problems.

We present a deterministic polynomial time reduction of the Discrete Logarithm Problem in $E_{p}$ to the classical Discrete Logarithm Problem in $\mathbb{Z}_{p}$, the $p$-element field. In particular, the Discrete Logarithm Problem in $E_{p}$ can be solved, by conventional computers, in subexponential time.
\end{abstract}

\section{INTRODUCTION}

For Discrete Logarithm based cryptography, it is desirable to find efficiently implementable groups for which sub-exponential algorithms for the Discrete Logarithm Problem are not available. Thus far, the only candidates for such groups seem to be (carefully chosen) groups of points on elliptic curves [5, 7]. Groups of invertible matrices over a finite field, proposed in [8, where proved by Menezes and $\mathrm{Wu}$ [6] inadequate for this purpose. Consequently, any candidate for a platform group for Discrete Logarithm based cryptography must not be efficiently embeddable in a group of matrices.

In 1974, Bergman proved that the ring $\operatorname{End}\left(\mathbb{Z}_{p} \times \mathbb{Z}_{p^{2}}\right)$ of endomorphisms of the group $\mathbb{Z}_{p} \times \mathbb{Z}_{p^{2}}$, where $p$ is a prime parameter, admits no embedding in any ring of matrices over a commutative ring [1]. In 2011, Climent, Navarro and Tortosa [3] described an efficient implementation of $E_{p}$ (reviewed below), proved that uniformly random elements of $E_{p}$ are invertible with probability greater than $1-2 / p$, and supplied an efficient way to sample the invertible elements of $E_{p}$ uniformly at random. Consequently, they proposed this ring as a potential source for intractable cryptographic problems. Climent et al. proposed a DiffieHellman type key exchange protocol over $E_{p}$, but it was shown by Kamal and Youssef 4 not to be related to the Discrete Logarithm Problem, and to be susceptible to a polynomial time attack.

We consider the Discrete Logarithm Problem in $E_{p}$. Since $E_{p}$ admits no embedding in any ring of matrices over a commutative ring, the Menezes-Wu reduction attack [6] is not directly applicable. We present, however, a deterministic polynomial time reduction of the Discrete Logarithm Problem in $E_{p}$ to the classical Discrete Logarithm Problem in $\mathbb{Z}_{p}$, the $p$-element field. In particular, the Discrete Logarithm Problem in $E_{p}$ can be solved by conventional computers in sub-exponential time, and $E_{p}$ offers no advantage, over $\mathbb{Z}_{p}$, for cryptography based on the Discrete Logarithm Problem. 


\section{Computing Discrete logarithms in $\operatorname{End}\left(\mathbb{Z}_{p} \times \mathbb{Z}_{p^{2}}\right)$}

Climent, Navarro and Tortosa [3] provide the following faithful representation of Bergman's Ring. The elements of $E_{p}$ are the matrices

$$
g=\left(\begin{array}{cc}
a & b \\
c p & v+u p
\end{array}\right), \quad a, b, c, u, v \in\{0, \ldots, p-1\} .
$$

Addition (respectively, multiplication) is defined by first taking ordinary addition (respectively, multiplication) over the integers, and then reducing each element of the first row modulo $p$, and each element of the second row modulo $p^{2}$. The ordinary zero and identity integer matrices serve as the additive and multiplicative neutral elements of $E_{p}$, respectively. The element $g$ is invertible in $E_{p}$ if and only if $a, v \neq 0$.

The group of invertible elements in a ring $R$ is denoted $R^{*}$. For an element $g$ in a group, $|g|$ denotes the order of $g$ in that group.

Definition 1. The Discrete Logarithm Problem in a ring $R$ is to find $x$ given an element $g \in R^{*}$ and its power $g^{x}$, where $x \in\{0,1, \ldots,|g|-1\}$.

Another version of the Discrete Logarithm Problem asks to find any $\tilde{x}$ such that $g^{\tilde{x}}=g^{x}$. The reductions given below are applicable, with minor changes, to this version as well, but it is known the two versions are essentially equivalent (see Appendix [B]).

By the standard amplification techniques, one can increase the success probability of any discrete logarithm algorithm with non-negligible success probability to become arbitrarily close to 1 . Thus, for simplicity, we may restrict attention to algorithms that never fail. For ease of digestion, we present our solution to the Discrete Logarithm Problem in $E_{p}$ by starting with the easier cases, and gradually building up. Not all of the easier reductions are needed for the main ones, but they do contain some of the important ingredients of the main ones, and may also be of independent interest to some readers.

\subsection{Basic reductions.}

Reduction 2. Computing the order of an element in $R^{*}$, using discrete logarithms in $R$.

Details. For $g \in R^{*}, g^{-1}=g^{|g|-1}$. Thus, $|g|=\log _{g}\left(g^{-1}\right)+1$.

Reduction 3. Computing discrete logarithms in a product of rings using discrete logarithms in each ring separately.

Details. For rings $R, S,(R \times S)^{*}=R^{*} \times S^{*}$. Let $(g, h) \in R^{*} \times S^{*}$ and $(g, h)^{x}=\left(g^{x}, h^{x}\right)$, where $x \in\{1, \ldots,|(g, h)|\}$, be given. Compute

$$
\begin{aligned}
& x \bmod |g|=\log _{g}\left(g^{x}\right) ; \\
& x \bmod |h|=\log _{h}\left(h^{x}\right) .
\end{aligned}
$$

Use Reduction 2 to compute $|g|$ and $|h|$. Compute, using the Chinese Remainder Algorithm,

$$
x \bmod \operatorname{lcm}(|g|,|h|)=x \bmod |(g, h)|=x .
$$

The Euler isomorphism is the function

$$
\begin{aligned}
\Phi_{p}:\left(\mathbb{Z}_{p},+\right) \times\left(\mathbb{Z}_{p}^{*}, \cdot\right) & \rightarrow \mathbb{Z}_{p^{2}}^{*} \\
(a, b) & \mapsto(1+a p) \cdot b^{p} \bmod p^{2} .
\end{aligned}
$$


The function $\Phi_{p}$ is easily seen to be an injective homomorphism between groups of equal cardinality, and thus an isomorphism of groups (cf. Paillier [9] in a slightly more involved context). The Euler isomorphism can be inverted efficiently: Given $c \in \mathbb{Z}_{p^{2}}^{*}$, let $a \in \mathbb{Z}_{p}, b \in \mathbb{Z}_{p}^{*}$ be such that $c=(1+a p) b^{p} \bmod p^{2}$. Then

$$
c=(1+a p) \cdot b^{p}=1 \cdot b^{p}=b \quad(\bmod p) .
$$

Compute $b=c \bmod p$, then $b^{p} \bmod p^{2}$, then $1+a p=c \cdot\left(b^{p}\right)^{-1} \bmod p^{2}$, where the inverse is in $\mathbb{Z}_{p^{2}}^{*}$. Since $1+a p<p^{2}$, we can subtract 1 and divide by $p$ to get $a$.

Reduction 4. Computing discrete logarithms in $\mathbb{Z}_{p^{2}}$ using discrete logarithms in $\mathbb{Z}_{p}$.

Details. Use the Euler isomorphism to transform the problem into a computation of a discrete logarithm in $\left(\mathbb{Z}_{p},+\right) \times\left(\mathbb{Z}_{p}^{*}, \cdot\right)$. Computing discrete logarithm in $\left(\mathbb{Z}_{p},+\right)$ is trivial. Apply Reduction 3 ,

\subsection{Algebraic lemmata.}

Definition 5. $\bar{E}_{p}$ is the ring of matrices $\left(\begin{array}{cc}a & b \\ p c & v\end{array}\right), a, b, c, v \in\{0,1, \ldots, p-1\}$, where addition and multiplication are carried out over $\mathbb{Z}$, and then entry $(2,1)$ is reduced modulo $p^{2}$, and the other three entries are reduced modulo $p$.

Lemma 6. The map

$$
\begin{aligned}
E_{p} & \rightarrow \bar{E}_{p} \\
\left(\begin{array}{cc}
a & b \\
c p & v+u p
\end{array}\right) & \mapsto\left(\begin{array}{cc}
a & b \\
c p & v
\end{array}\right)
\end{aligned}
$$

is a ring homomorphism.

Proof. Since addition is component-wise, it remains to verify multiplicativity. Indeed, in $E_{p}$,

$$
\left(\begin{array}{cc}
a_{1} & b_{1} \\
c_{1} p & v_{1}+u_{1} p
\end{array}\right)\left(\begin{array}{cc}
a_{2} & b_{2} \\
c_{2} p & v_{2}+u_{2} p
\end{array}\right)=\left(\begin{array}{cc}
a_{1} a_{2} & a_{1} b_{2}+b_{1} v_{2} \\
\left(c_{1} a_{2}+v_{1} c_{2}\right) p & v_{1} v_{2}+\left(c_{1} b_{2}+v_{1} u_{2}+u_{1} v_{2}\right) p
\end{array}\right)
$$

and in $\bar{E}_{p}$,

$$
\left(\begin{array}{cc}
a_{1} & b_{1} \\
c_{1} p & v_{1}
\end{array}\right)\left(\begin{array}{cc}
a_{2} & b_{2} \\
c_{2} p & v_{2}
\end{array}\right)=\left(\begin{array}{cc}
a_{1} a_{2} & a_{1} b_{2}+b_{1} v_{2} \\
\left(c_{1} a_{2}+v_{1} c_{2}\right) p & v_{1} v_{2}
\end{array}\right)
$$

Lemma 7. Let $\bar{g}=\left(\begin{array}{cc}a & b \\ c p & v\end{array}\right) \in \bar{E}_{p}^{*}$, and let $x$ be a natural number. Define $d_{x} \in \mathbb{Z}_{p}$ by

$$
d_{x}= \begin{cases}\frac{a^{x}-v^{x}}{a-v} & a \neq v \\ x a^{x-1} & a=v\end{cases}
$$

Then

$$
\bar{g}^{x}=\left(\begin{array}{cc}
a^{x} & b d_{x} \\
c d_{x} p & v^{x}
\end{array}\right)
$$


Proof. By induction on $x$. The statement is immediate when $x=1$. Induction step: If $a \neq v$, then in $\mathbb{Z}_{p}$,

$$
\begin{aligned}
& a^{x}+d_{x} v=a^{x}+\frac{a^{x}-v^{x}}{a-v} \cdot v=\frac{a^{x}(a-v)+\left(a^{x}-v^{x}\right) v}{a-v}=\frac{a^{x+1}-v^{x+1}}{a-v}=d_{x+1} ; \\
& a d_{x}+v^{x}=\frac{a\left(a^{x}-v^{x}\right)}{a-v}+\frac{(a-v) v^{x}}{a-v}=\frac{a^{x+1}-v^{x+1}}{a-v}=d_{x+1} .
\end{aligned}
$$

If $a=v$, then

$$
\begin{aligned}
& a^{x}+d_{x} v=a^{x}+x a^{x-1} v=a^{x}+x a^{x-1} a=a^{x}+x a^{x}=(x+1) a^{x}=d_{x+1} ; \\
& a d_{x}+v^{x}=x a^{x}+a^{x}=(x+1) a^{x}=d_{x+1} .
\end{aligned}
$$

Thus, in either case,

$$
\bar{g}^{x+1}=\bar{g}^{x} \cdot \bar{g}=\left(\begin{array}{cc}
a^{x} & b d_{x} \\
c d_{x} p & v^{x}
\end{array}\right) \cdot\left(\begin{array}{cc}
a & b \\
c p & v
\end{array}\right)=\left(\begin{array}{cc}
a^{x+1} & b\left(a^{x}+d_{x} v\right) \\
c\left(a d_{x}+v^{x}\right) p & v^{x+1}
\end{array}\right)=\left(\begin{array}{cc}
a^{x+1} & b d_{x+1} \\
c d_{x+1} p & v^{x+1}
\end{array}\right) .
$$

Lemma 8. Let $\bar{g}=\left(\begin{array}{cc}a & b \\ c p & v\end{array}\right) \in \bar{E}_{p}^{*}$.

(1) If $a=v$ and at least one of $b, c$ is nonzero, then $|\bar{g}|=p \cdot|a|$.

(2) In all other cases $(a \neq v$ or $b=c=0),|\bar{g}|=\operatorname{lcm}(|a|,|v|)$.

Proof. Define $d_{x}$ as in Lemma 7. By Lemma 7.

$$
\left(\begin{array}{cc}
a^{|\bar{g}|} & * \\
* & v^{|\bar{g}|}
\end{array}\right)=\bar{g}^{|\bar{g}|}=\left(\begin{array}{cc}
1 & 0 \\
0 & 1
\end{array}\right) .
$$

Thus, $|a|$ and $|v|$ divide $|\bar{g}|$, and therefore so does $\operatorname{lcm}(|a|,|v|)$.

We consider all possible cases.

If $b=c=0$, then

$$
\bar{g}^{x}=\left(\begin{array}{cc}
a^{x} & 0 \\
0 & v^{x}
\end{array}\right)
$$

for all $x$, and thus $|\bar{g}|=\operatorname{lcm}(|a|,|v|)$, as claimed in (2).

Assume, henceforth, that at least one of $b, c$ is nonzero, and let

$$
l=\operatorname{lcm}(|a|,|v|) .
$$

If $a \neq v$, then

$$
d_{l}=\frac{a^{l}-v^{l}}{a-v}=\frac{1-1}{a-v}=0 \quad \bmod p,
$$

and thus, by Lemma $4, \bar{g}^{l}=I$. Thus, $|\bar{g}|$ divides $l$, which we have seen to divide $|\bar{g}|$. It follows that $|\bar{g}|=l$, as claimed in (2).

Assume, henceforth, that $a=v$.

Since $d_{p}=p a^{p-1}=0 \bmod p$, we have by Lemma 7 that

$$
\bar{g}^{p}=\left(\begin{array}{cc}
a^{p} & 0 \\
0 & a^{p}
\end{array}\right)=\left(\begin{array}{ll}
a & 0 \\
0 & a
\end{array}\right) .
$$


It follows that $\bar{g}^{p \cdot|a|}=I$. Therefore, $|\bar{g}|$ divides $p \cdot|a|$. Recall that $|a|$ divides $|\bar{g}|$. Now, $d_{|a|}=|a| \cdot a^{|a|-1} \bmod p$. Since $|a|<p, d_{|a|} \neq 0$. It follows that

$$
\bar{g}^{|a|}=\left(\begin{array}{cc}
a^{|a|} & b d_{|a|} \\
c d_{|a|} p & a^{|a|}
\end{array}\right) \neq\left(\begin{array}{cc}
1 & 0 \\
0 & 1
\end{array}\right)
$$

and thus $|\bar{g}|=p \cdot|a|$, as claimed in (1).

\subsection{The main reductions.}

Reduction 9. Computing discrete logarithms in $\bar{E}_{p}$ using discrete logarithms in $\mathbb{Z}_{p}$.

Details. Let $\bar{g}=\left(\begin{array}{cc}a & b \\ c p & v\end{array}\right) \in \bar{E}_{p}^{*}$, and let $x \in\{1, \ldots,|\bar{g}|\}$. By Lemma [7,

$$
\bar{g}^{x}=\left(\begin{array}{cc}
a^{x} & b d_{x} \\
c d_{x} p & v^{x}
\end{array}\right) .
$$

If $a \neq v$ or $b=c=0$, then by Lemma $8,|\bar{g}|=\operatorname{lcm}(|a|,|v|)$. Compute

$$
\begin{aligned}
x \bmod |a| & =\log _{a}\left(a^{x}\right) ; \\
x \bmod |v| & =\log _{v}\left(v^{x}\right) .
\end{aligned}
$$

Since $x<|\bar{g}|$, we can use the Chinese Remainder Algorithm to compute $x \bmod \operatorname{lcm}(|a|,|v|)=$ $x$.

Thus, assume that $a=v$ and one of $b, c$ is nonzero. By Lemma $8,|\bar{g}|=p \cdot|a|$. Compute

$$
x_{0}:=x \bmod |a|=\log _{a}\left(a^{x}\right) .
$$

Compute

$$
\bar{g}^{x} \cdot \bar{g}^{-x_{0}}=\bar{g}^{x-x_{0}}=\left(\begin{array}{cc}
a^{x-x_{0}} & b d_{x-x_{0}} \\
c d_{x-x_{0}} p & a^{x-x_{0}}
\end{array}\right)=\left(\begin{array}{cc}
1 & b d_{x-x_{0}} \\
c d_{x-x_{0}} p & 1
\end{array}\right) .
$$

Since $b$ or $c$ is nonzero, we can extract $d_{x-x_{0}} \bmod p$. Compute

$$
d_{x-x_{0}} \cdot a=\left(x-x_{0}\right) a^{x-x_{0}}=x-x_{0} \bmod p .
$$

As $x-x_{0} \leq x<|\bar{g}|=p \cdot|a|$, we can use the Chinese Remainder Algorithm to compute

$$
x-x_{0} \bmod \operatorname{lcm}(p,|a|)=x-x_{0} \bmod p \cdot|a|=x-x_{0} .
$$

Add $x_{0}$ to obtain $x$.

Reduction 10. Computing discrete logarithms in $E_{p}$ using discrete logarithms in $\mathbb{Z}_{p}$.

Details. Let $g=\left(\begin{array}{cc}a & b \\ c p & v+u p\end{array}\right) \in E_{p}^{*}$, and let $x \in\{1, \ldots,|g|\}$. Take $\bar{g}=\left(\begin{array}{cc}a & b \\ c p & v\end{array}\right) \in \bar{E}_{p}^{*}$. Use Lemma 8 and Reduction 2 to compute $|\bar{g}|$. By Lemma 6 , $|\bar{g}|$ divides $|g|$. As $\bar{g}^{|\bar{g}|}=I$ is the image of $g^{|\bar{g}|}$ under the homomorphism of Lemma 6. we have that

$$
g^{|\bar{g}|}=\left(\begin{array}{cc}
1 & 0 \\
0 & 1+s p
\end{array}\right)
$$

for some $s \in\{0, \ldots, p-1\}$. Using Reduction 9, compute

$$
x_{0}:=\log _{\bar{g}}\left(\bar{g}^{x}\right)=x \bmod |\bar{g}| \text {. }
$$


If $s=0$ then $|g|=|\bar{g}|$, and thus $x_{0}:=\log _{\bar{g}}\left(\bar{g}^{x}\right)=\log _{g}\left(g^{x}\right)=x$, and we are done. If $s \neq 0$, let $q=\left(x-x_{0}\right) /|\bar{g}|$. Since the order of $1+s p$ in $\mathbb{Z}_{p^{2}}$ is $p$ (in $\mathbb{Z}_{p^{2}},(1+s p)^{e}=1+e s p$ for all $e$ ), the order of $g^{|\bar{g}|}$ is $p$, and thus $|g|=|\bar{g}| \cdot p$. Thus, $q \leq x /|\bar{g}|<|g| /|\bar{g}|=p$. Compute

$$
g^{x} g^{-x_{0}}=g^{x-x_{0}}=\left(g^{|\bar{g}|}\right)^{q}=\left(\begin{array}{cc}
1 & 0 \\
0 & 1+s p
\end{array}\right)^{q}=\left(\begin{array}{cc}
1 & 0 \\
0 & (1+s p)^{q}
\end{array}\right)=\left(\begin{array}{cc}
1 & 0 \\
0 & 1+s q p
\end{array}\right) .
$$

Compute $s q \bmod p=((1+s q p)-1) / p$. In $\mathbb{Z}_{p}$, multiply by $s^{-1}$ to obtain $q \bmod p=q$. Multiply by $|\bar{g}|$ to get $x-x_{0}$, and add $x_{0}$.

\section{Summing up: Code}

Following is a self-explanatory code (in Magma [2]) of our main reductions. This code shows, in a concise manner, that the number of computations of discrete logarithms in $\mathbb{Z}_{p}$ needed to compute discrete logarithms in Bergman's Ring $E_{p}$ is at most 2. For completeness, we provide, in Appendix A, the basic routines.

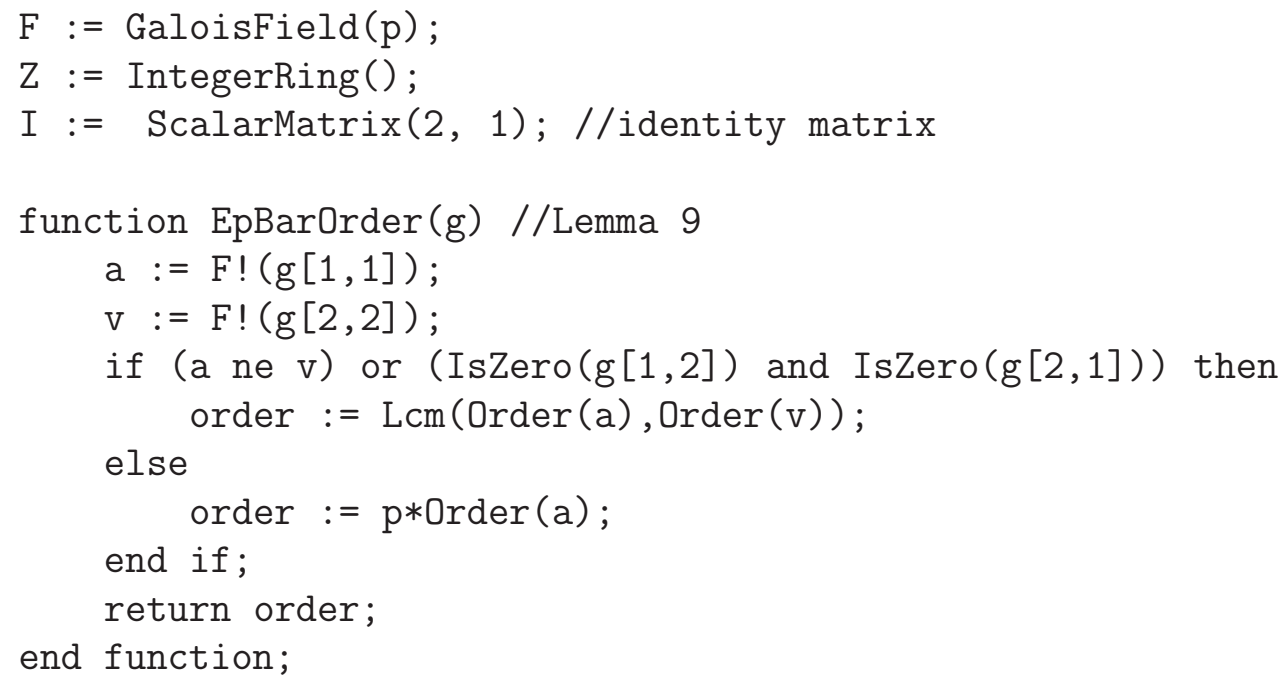




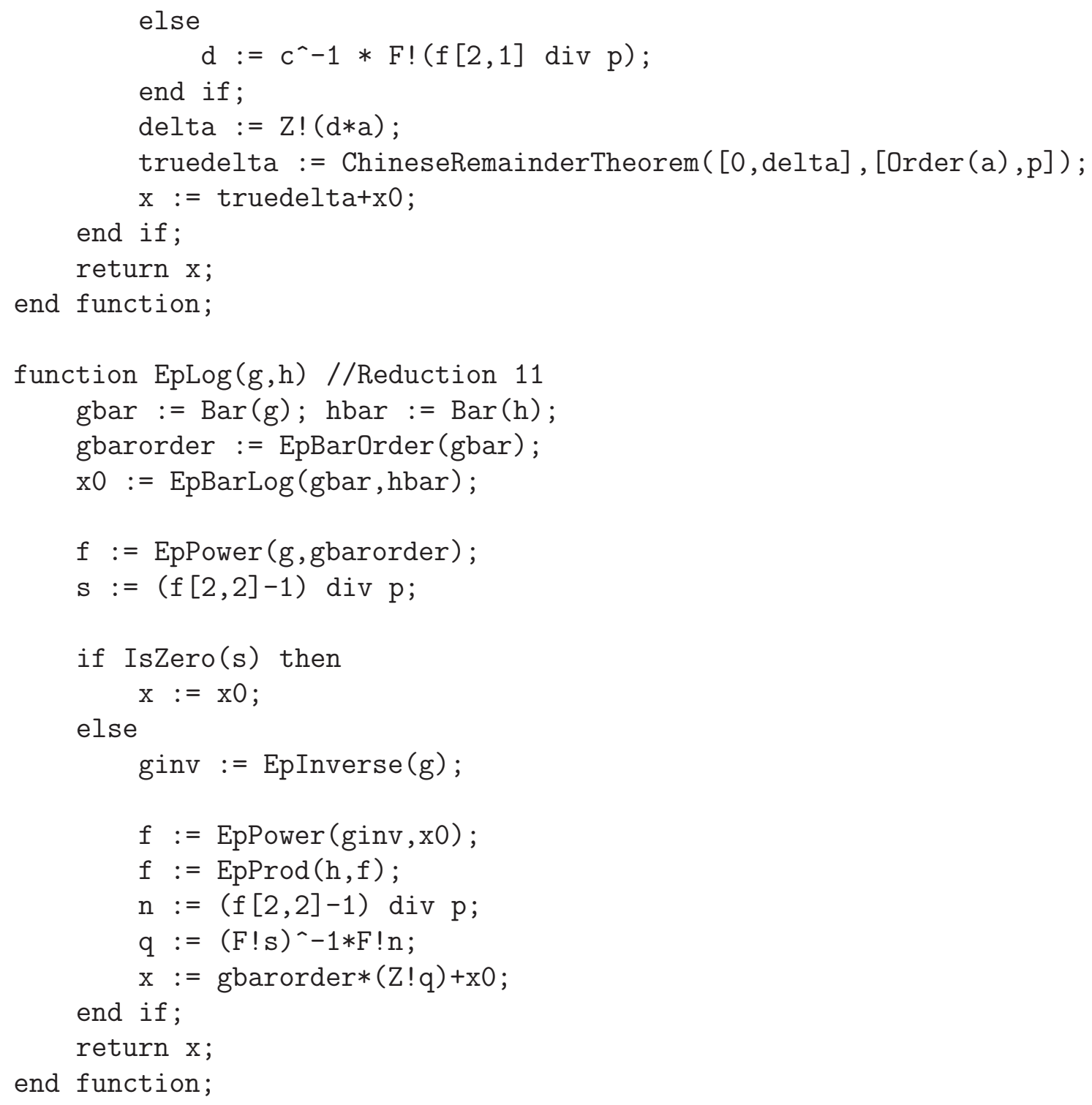

We have tested these routines extensively: For random primes of size 4,8,16,32,64, and 128 bits, and thousands of random pairs $g, h=g^{x}, \operatorname{EpLog}(g, h)$ always returned $x$.

\section{REFERENCES}

[1] G. Bergman, Examples in PI ring theory, Israel Journal of Mathematics 18 (1974), 257-277.

[2] W. Bosma, J. Cannon, and C. Playoust, The Magma algebra system, I: The user language, Journal of Symbolic Computation 24 (1997), 235-265.

[3] J. Climent, P. Navarro, L. Tortosa, On the arithmetic of the endomorphisms ring $\operatorname{End}\left(\mathbb{Z}_{p} \times \mathbb{Z}_{p^{2}}\right)$, Applicable Algebra in Engineering, Communication and Computing 22 (2011), 91-108.

[4] A. Kamal, A. Youssef, Cryptanalysis of a key exchange protocol based on the endomorphisms ring End $\left(\mathbb{Z}_{p} \times \mathbb{Z}_{p^{2}}\right)$, Applicable Algebra in Engineering, Communication and Computing, to appear.

[5] N. Koblitz, Elliptic curve cryptosystems, Mathematics of Computation 48 (1987), 203-209.

[6] A. Menezes, Y. Wu, The discrete logarithm problem in GL(n,q), Ars Combinatoria, 47 (1998), 23-32.

[7] V. Miller, Uses of elliptic curves in cryptography, in: Advances in Cryptology-Proceedings of Crypto '85. Lecture Notes in Computer Science 218 (1986), 417-426. 
[8] R. Odoni, R. Sanders, V. Varadharajan, Public key distribution in matrix rings, Electronic Letters 20 (1984), 386-387.

[9] P. Paillier, Public-key cryptosystems based on composite degree residuosity classes, in: J. Stern, ed., Advances in Cryptology - EUROCRYPT'99, Lecture Notes in Computer Science 1592 (1999), 223238.

\section{Appendix A. Elementary routines}

To remove any potential ambiguity, and help readers interested in reproducing our experiments, we provide here the basic routines for arithmetic in Bergman's Ring $E_{p}$.

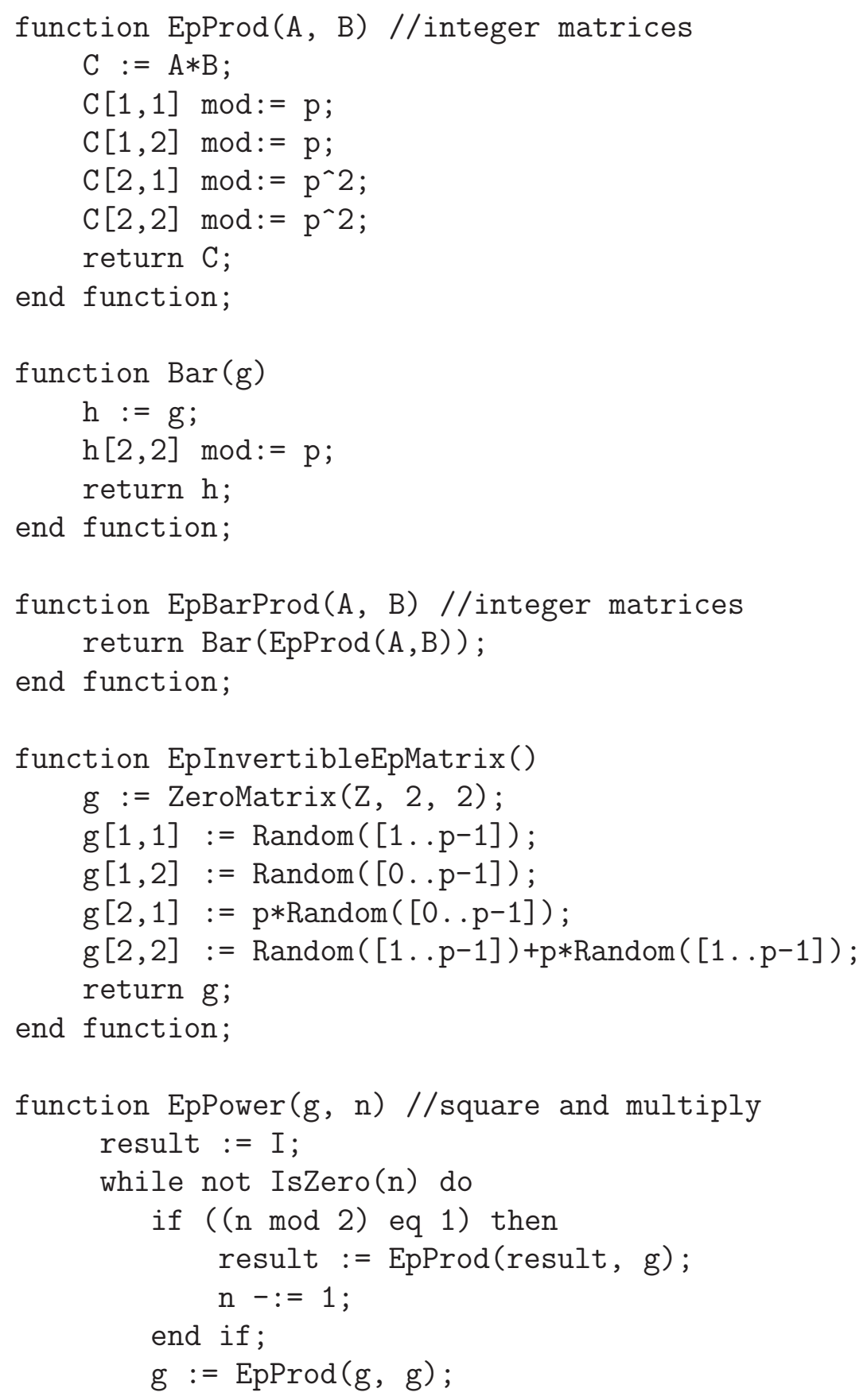




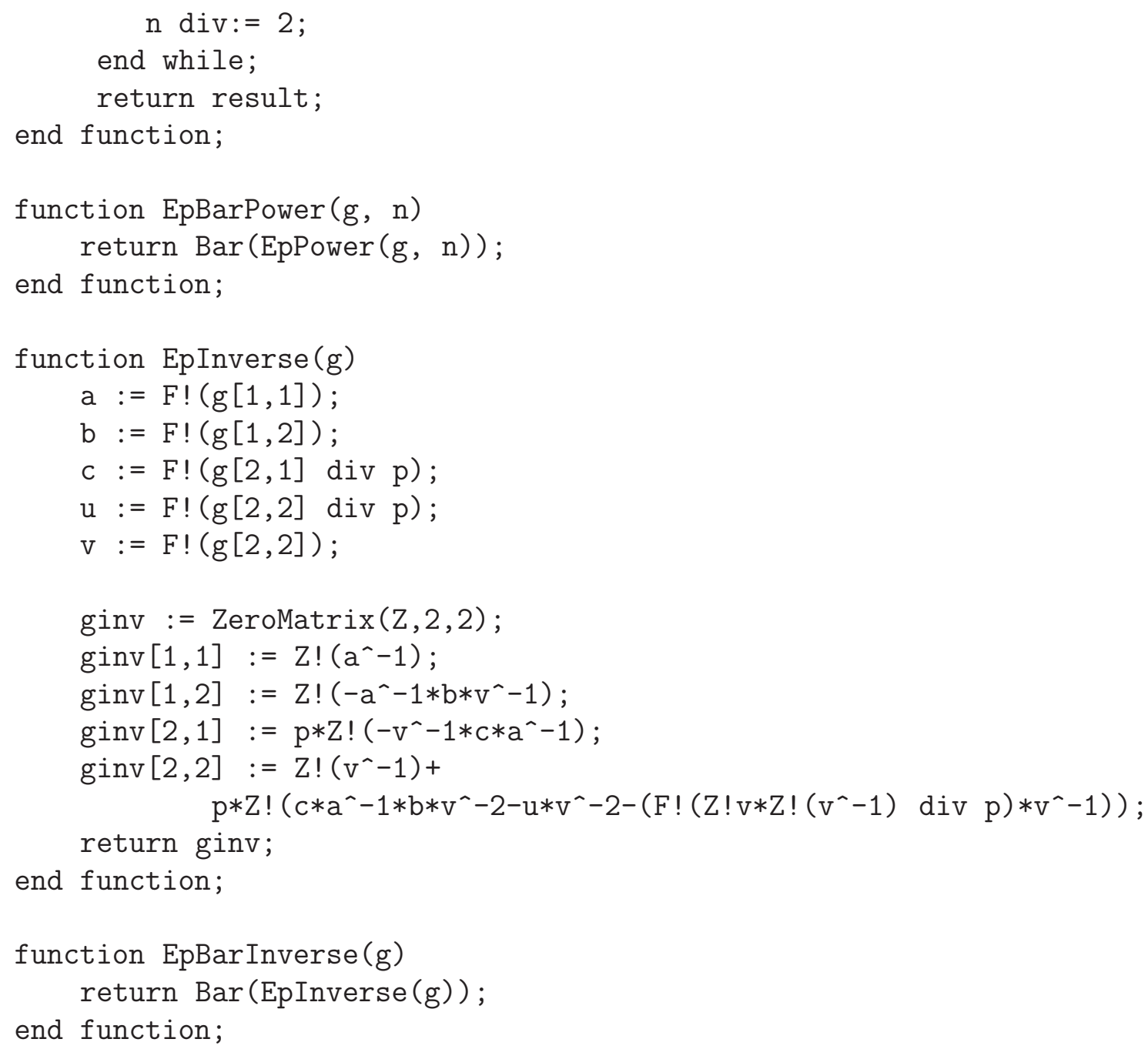

\section{Appendix B. Equivalence of Discrete Logarithm Problems}

The result in this appendix should be well known to experts, but since we are not aware of any reference for it, we include it for completeness. Consider the following two versions of the Discrete Logarithm Problem in a prescribed finite group $G$. We assume that $|G|$, or a polynomial upper bound $K$ on $|G|$, is known. We do not assume that $G$ is cyclic.

DLP1: Find $x$, given an element $g \in G$ and its power $g^{x}$, where $x \in\{0,1, \ldots,|g|-1\}$. DLP2: Given an element $g \in G$ and its power $g^{x}$, find $\tilde{x}$ with $g^{\tilde{x}}=g^{x}$.

DLP1 is harder than DLP2: A DLP1 oracle returns $\tilde{x}:=x \bmod |g|$ on input $g, g^{x}$. On the other hand, DLP2 is probabilistically harder than DLP1: It suffices to show how $|g|$ can be computed using a DLP2 oracle. Indeed, for a large enough (but polynomial) number of random elements $r \in\{K, K+1, \ldots, M\}$ where $M \gg K$ is fixed, let $\tilde{r}$ be the output of DLP2 
on $\left(g, g^{r}\right)$. Then $|g|$ divides all numbers $(r-\tilde{r}) \bmod g$, and the greatest common divisor of these numbers is $|g|$, except for a negligible probability.

Department of Mathematics, Bar-Ilan University, Ramat Gan 52900, Israel

E-mail address: baninmmm@gmail.com, tsaban@math.biu.ac.il

$U R L:$ http://www.cs.biu.ac.il/〜tsaban 